\title{
NSAID enteropathy and bacteria: a complicated relationship
}

\author{
Stephanie D. Syer - Rory W. Blackler - Rebeca Martin - Giada de Palma • \\ Laura Rossi - Elena Verdu • Premek Bercik • Michael G. Surette • \\ Anne Aucouturier $\cdot$ Philippe Langella $\cdot$ John L. Wallace
}

Received: 1 December 2014 / Accepted: 5 December 2014/Published online: 10 January 2015

(C) Springer Japan 2015

\begin{abstract}
The clinical significance of small intestinal damage caused by nonsteroidal anti-inflammatory drugs (NSAIDs) remains under-appreciated. It occurs with greater frequency than the damage caused by these drugs in the upper gastrointestinal tract, but is much more difficult to diagnose and treat. Although the pathogenesis of NSAID enteropathy remains incompletely understood, it is clear that bacteria, bile, and the enterohepatic circulation of NSAIDs are all important factors. However, they are also interrelated with one another. Bacterial enzymes can affect the cytotoxicity of bile and are essential for enterohepatic circulation of NSAIDs. Gram-negative bacteria appear to be particularly important in the pathogenesis of NSAID
\end{abstract}

Part of this review was presented at The 4th International Forum of the 100th General Meeting of the Japanese Society of Gastroenterology.

Stephanie D. Syer and Rory W. Blackler contributed equally to this article.

S. D. Syer · R. W. Blackler · G. de Palma - L. Rossi ·

E. Verdu · P. Bercik · M. G. Surette

Department of Medicine, McMaster University, Hamilton,

Canada

R. Martin · A. Aucouturier · P. Langella

INRA Micalis, Jouy-en-Josas, France

J. L. Wallace $(\square)$

Department of Physiology and Pharmacology,

University of Calgary, 3330 Hospital Drive NW, Calgary,

AB T2N 4N1, Canada

e-mail: wallacej@ucalgary.ca

\section{J. L. Wallace}

Department of Pharmacology and Toxicology,

University of Toronto, 15 Prince Arthur Avenue,

Toronto, ON M5R 1B2, Canada enteropathy, possibly through release of endotoxin. Inhibitors of gastric acid secretion significantly aggravate NSAID enteropathy, and this effect is due to significant changes in the intestinal microbiome. Treatment with antibiotics can, in some circumstances, reduce the severity of NSAID enteropathy, but published results are inconsistent. Specific antibiotic-induced changes in the microbiota have not been causally linked to prevention of intestinal damage. Treatment with probiotics, particularly Bifidobacterium, Lactobacillus, and Faecalibacteriaum prausnitzii, has shown promising effects in animal models. Our studies suggest that these beneficial effects are due to colonization by the bacteria, rather than to products released by the bacteria.

Keywords Inflammation - Ulcer · Microbiota - Intestine * Bleeding $\cdot$ Hydrogen sulfide $\cdot$ Probiotics $\cdot$ Antibiotics

\section{NSAID enteropathy: an under-recognized clinical problem}

Nonsteroidal anti-inflammatory drugs (NSAIDs) remain among the most widely used drugs worldwide despite significant adverse effects that include gastrointestinal (GI) ulceration and bleeding $[1,2]$ and an increased risk of serious cardiovascular events [3]. Although there are drugs available for preventing the upper GI tract adverse effects of NSAIDs (inhibitors of gastric acid secretion), no measures have been identified that have been proven to be effective at preventing the small intestinal injury caused by these drugs $[1,4,5]$. There are also no proven-effective treatments for NSAID enteropathy once it has occurred $[1,2,4]$. With improved means of detection, such as video capsule endoscopy, physicians are becoming more aware 
that the small intestinal damage caused by NSAIDs is much more common and much more serious than previously recognized [4, 6-11]. The prevalence of NSAIDinduced damage in the small intestine was highlighted by data showing that various features consistent with damage are common among long-term NSAID users: inflammation in 60-70 \%, ulceration in 30-40\%, increased permeability in up to $70 \%$, bleeding/anemia in $30 \%$, and malabsorption in $40-70 \%$ [4]. These authors pointed out that "the magnitude of these serious outcomes from the lower GI tract is not well defined, but recent data suggest that they may be as frequent and severe as upper GI complications" [4]. McCarthy [10] expressed a similar view, pointing out that in the VIGOR trial [12], most of the damage and bleeding caused by naproxen and rofecoxib occurred in the small intestine, distal to the ligament of Treitz.

Unfortunately, the cost of video capsule endoscopy has limited its widespread use, so NSAID enteropathy continues to be underdiagnosed. There is a lack of simple, noninvasive methods with sufficient sensitivity and specificity for detecting ulceration and bleeding in the small intestine. An additional problem with detecting NSAID enteropathy is that patients often do not have symptoms that would alert them or their physician to a problem. Moreover, as compared with upper GI tract events requiring hospitalization, lower GI tract events were associated with higher rates of mortality and relapse, and significantly longer periods of hospitalization [11].

\section{A relationship between acid suppression and NSAID enteropathy?}

In 2009, Lanas et al. [11] reported that hospitalizations due to lower GI tract adverse events had been steadily rising during the 1990s and the first decade of this century, when use of proton pump inhibitors (PPIs) and selective cyclooxygenase 2 inhibitors became prevalent. During the same period, hospitalizations due to upper GI tract adverse events declined significantly. The decrease in the incidence of upper GI events was easy to understand, but why was the incidence of lower GI tract events increasing? We wondered if the rise in lower GI tract events might be mechanistically linked to the increased use of PPIs. In 2011, we reported that in rodents, suppression of acid secretion with PPIs, although effective at preventing gastroduodenal damage, resulted in a dramatic worsening of distal intestinal damage [13]. This was confirmed by others, and was shown to occur also with histamine $\mathrm{H}_{2}$ receptor antagonists [14-16]. Moreover, there is clinical evidence linking use of PPIs and $\mathrm{H}_{2}$ receptor antagonists to severe intestinal damage observed in rheumatoid arthritis patients taking NSAIDs [8]. We also observed that low- dose aspirin significantly exacerbated NSAID-induced small intestinal ulceration and bleeding [13].

The mechanism underlying the increase in intestinal damage in rats treated with a PPI was linked to a dramatic change in the intestinal microbiota. In particular, there was a substantial decrease in the numbers of Bifidobacterium in the intestine following PPI administration [13]. Restoration of intestinal Bifidobacterium numbers resulted in a reversal of the exacerbation of intestinal injury by PPIs. Studies performed in germ-free mice confirmed that susceptibility to NSAID-induced small intestinal injury could be transferred via the microbiota [13].

Many studies have implicated bacteria in the pathogenesis of NSAID enteropathy, but the underlying mechanism has not been identified. Bacteria will rapidly colonize ulcers, and can delay their healing [17], but in terms of a primary role in ulceration, bacteria may contribute through effects on the cytotoxicity of bile. Secondary bile acids are particularly damaging to epithelial cells [18]. The conversion of primary bile acids to secondary bile acids is catalyzed by bacterial enzymes [19]. Treatment of rats with PPIs or $\mathrm{H}_{2}$ receptor antagonists has been shown to markedly increase the cytotoxicity of bile [20]. The presence of NSAIDs in bile (free or glucuronidated) may also contribute to epithelial damage and subsequent ulceration [21]. Enterohepatic circulation of NSAIDs is key to the induction of intestinal damage [2225 ], and is dependent on bacterial $\beta$-glucuronidases [26]. Inhibition of $\beta$-glucuronidase activity has been shown to prevent NSAID enteropathy in mice [26]. Germ-free rats do not develop intestinal damage when given NSAIDs, further suggesting a key role of bacteria in the pathogenesis of this injury $[27,28]$. However, this could be related to the absence of bacterial enzymes important to the processes outlined above (e.g., conversion of primary bile acids to secondary bile acids and deglucuronidation to allow reabsorption of NSAIDs in the ileum and their subsequent enterohepatic circulation).

\section{Do antibiotics prevent NSAID enteropathy?}

Although antibiotics have been suggested as a viable approach to prevention of NSAID enteropathy, there is very little clinical evidence to support such use. For example, metronidazole was suggested to be effective for such an application, but this was based on a single, openlabel study [29]. On the other hand, there have been numerous studies on the effects of antibiotics in animal models of NSAID enteropathy. One of the consistent findings from animal studies of the role of bacteria in NSAID enteropathy has been the increase in Gram-negative bacteria in the intestine following NSAID 
administration [13, 23, 30]. A role for Gram-negative bacteria in the pathogenesis of NSAID enteropathy is supported by evidence that bacterial endotoxin contributes significantly to ulceration in the intestine [31]. Koga et al. [32] reported that oral pretreatment of rats with kanamycin, which targets Gram-negative bacteria, dose-dependently prevented the small intestinal injury induced by indomethacin. Leite et al. [33] reported protective effects against indomethacin-induced enteropathy by metronidazole, which is effective at killing Gram-negative and Gram-positive anaerobes. However, these treatments have not been found to be consistently effective [30]. For example, as shown in Fig. 1, we observed that treatment of rats with kanamycin had no significant effect on naproxeninduced small intestinal damage. In the same study, treatment with vancomycin (which mainly targets Gram-positive bacteria) or rifaximin (a broad-spectrum, nonabsorbed antibiotic) also failed to significantly affect the severity of naproxen-induced enteropathy. In each of these experiments, the rats received the antibiotic for several days prior to initiation of twice daily naproxen administration.

In contrast to these studies of a single antibiotic, studies in which a cocktail of antibiotics was used have consistently demonstrated a protective effect against NSAIDinduced intestinal damage $[23,28,30]$. For example, Kent et al. [23] observed a significant reduction of indomethacin-induced intestinal damage with a cocktail of antibiotics (neomycin, polymixin B, and bacitracin). Similarly, we observed (Fig. 2) that treatment with a cocktail of ampicillin, vancomycin, neomycin, and metronidazole resulted in a significant diminution of the severity of naproxeninduced intestinal damage. Despite treatment with the antibiotic cocktail, in the drinking water, for several days prior to treatment with the NSAID, the intestinal damage was reduced, but not prevented. This is consistent with the early observations of Kent et al. [23], who remarked: "since these agents do not prevent completely the ulceration, we think that these agents reduce the severity of the lesions by allowing healing to start sooner, rather than by preventing some synergistic effect between the flora and the drug in causing the initial damage". GI ulcers are rapidly colonized by bacteria (even in the near-sterile stomach), and colonization generally delays ulcer healing, although healing can be promoted through selective colonization of the ulcers with certain bacteria [17].

To try to gain a better understanding of the mechanisms through which antibiotics may affect susceptibility to NSAID enteropathy, we examined the changes in microbiota in rats treated with different antibiotics by partial $16 \mathrm{~S}$ RNA gene profiling using Illumina sequencing of cecal samples from each rat [34]. As shown in Fig. 3, the intestinal microbiome of healthy rats was dominated by bacteria of the phyla Firmicutes and Bacteroidetes.
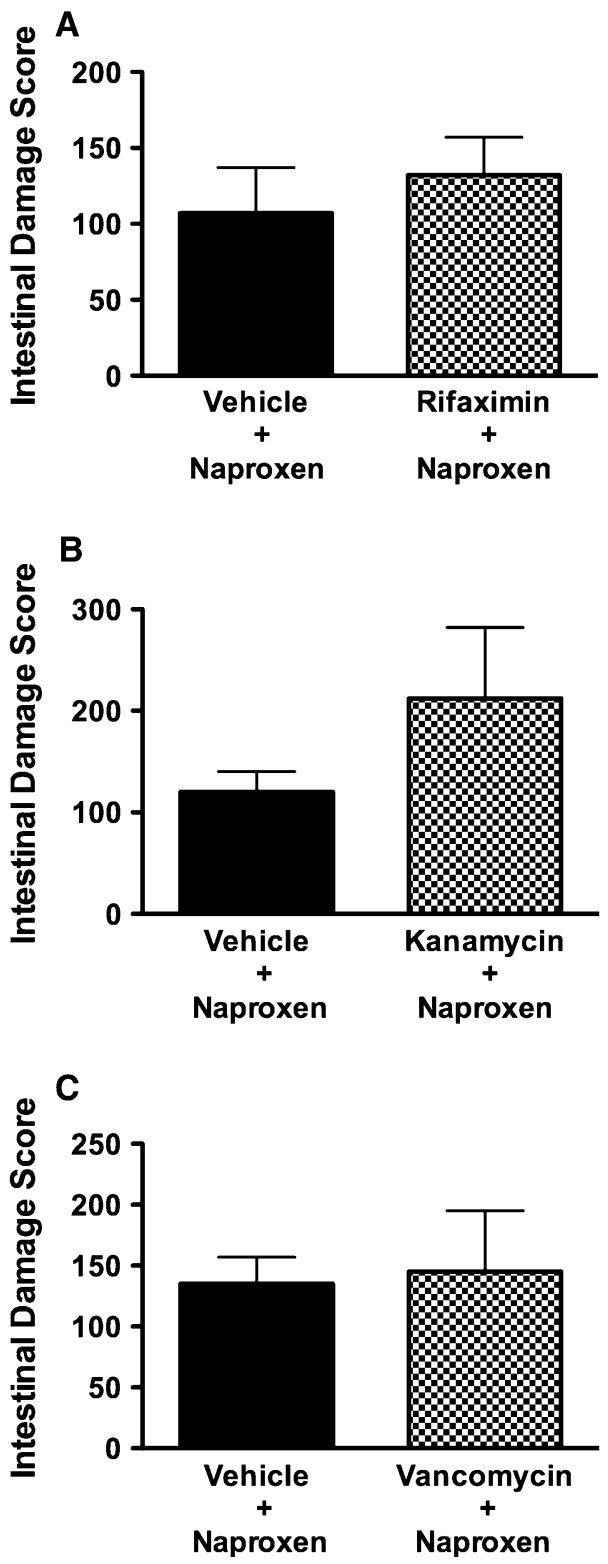

Fig. 1 When administered to rats once daily for 5 days prior to initiating twice daily naproxen treatment, rifaximin (a), kanamycin (b), and vancomycin (c) did not significantly affect the severity of intestinal damage. The entire small intestine was examined by an individual blind to the treatments the rats received. The area of each ulcer was measured with calipers, and was summed to give the "intestinal damage score". Each bar represents the mean \pm the standard error of the mean of at least six rats per group. The damage scores for antibiotic-treated groups did not differ significantly from the damage scores for vehicle-treated groups (Student's $t$ test)

Administration of naproxen over several days did not appear to cause much of a change in the microbiota, but actually there were significant shifts in the nondominant species (in particular, a marked increase in Proteobacteria), and these changes may contribute to intestinal damage. Vancomycin is an antibiotic that primarily targets Gram-positive bacteria, so not surprisingly, in rats treated 


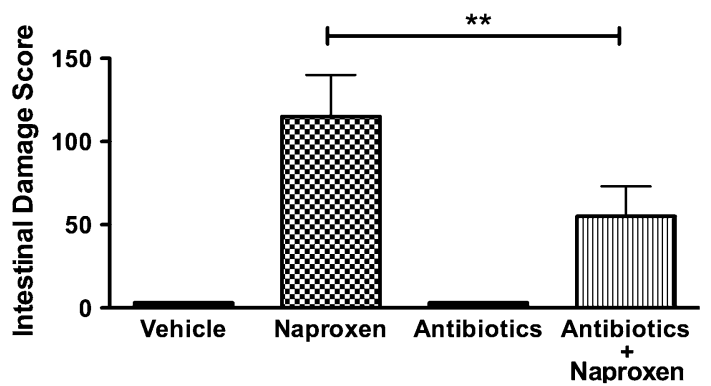

Fig. 2 Treatment of rats with a cocktail of antibiotics, added to the drinking water, significantly reduced the severity of naproxeninduced small intestinal ulceration. The antibiotic cocktail consisted of ampillicin sodium salt $(1 \mathrm{~g} / \mathrm{L})$, vancomycin hydrochloride $(500 \mathrm{mg} / \mathrm{L})$, neomycin trisulfate salt hydrate $(1 \mathrm{~g} / \mathrm{L})$, and metronidazole $(1 \mathrm{~g} / \mathrm{L})$. It was provided to the rats for a total of 9 days, and naproxen (or vehicle) was administered twice daily over the final 4 days. Water consumption in the four groups of rats did not differ significantly during the study. Naproxen was given at $20 \mathrm{mg} / \mathrm{kg}$. The results are shown at the mean \pm the standard error of the mean of at least six rats per group. ${ }^{*} p<0.01$ (one-way ANOVA and Dunnett's multiple comparison test)

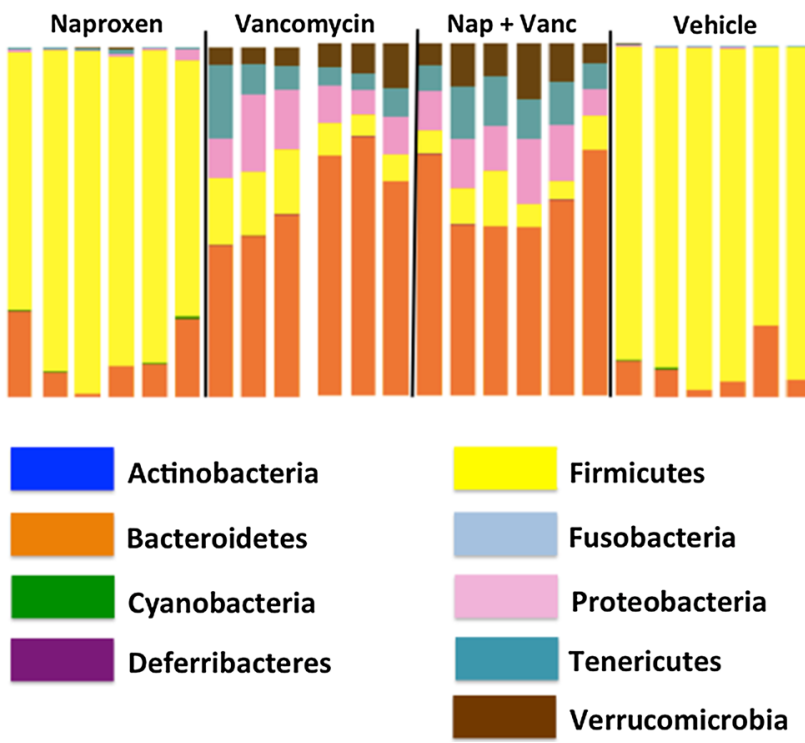

Fig. 3 Treatment with naproxen $(20 \mathrm{mg} / \mathrm{kg})$ and vancomycin $(50 \mathrm{mg} / \mathrm{kg})$ caused significant shifts in the microbiome of rats. Groups of 12 rats were treated orally with vehicle or vancomycin for a total of 9 days. Subgroups of six rats were treated orally with naproxen or vehicle twice daily for the final 4 days. 16S RNA Illumina sequencing of cecal samples from each rat was performed. Each vertical bar represents the sequencing data for one rat. Treatment with vehicle or vancomycin did not elicit any intestinal damage, whereas administration of naproxen plus vehicle or naproxen plus vancomycin caused extensive intestinal damage (mean scores of 135 and 145, respectively; see Fig. 1c). Relative to the vehicle treatment, naproxen administration caused an increase in the levels of Gram-negative bacteria, but those changes appeared small in comparison with the effects of vancomycin, which caused a shift from predominantly Gram-positive bacteria (Firmicutes) to a much greater propensity of various Gram-negative bacteria (Verrucomicrobia, Fusobacteria, Proteobacteria, Tenericutes). Nap naproxen, Vanc vancomycin with this agent there was a marked diminution of the levels of Firmicutes (Gram-positive bacteria) and a marked increase in Gram-negative bacteria (e.g., Bacteroidetes, Tenericutes, Proteobacteria, Verrucomicrobia). By itself, vancomycin did not cause intestinal damage. However, when naproxen was administered to vancomycin-treated rats, there was extensive intestinal damage, comparable in severity to that observed with naproxen alone (see Fig. 1c). The microbiota profile produced by vancomycin was largely preserved when naproxen was coadministered (Fig. 3).

Figure 4 shows the results of Illumina sequencing of cecal samples from rats treated with kanamycin. Kanamycin also markedly reduced Firmicutes levels, although not to the same extent as did vancomycin. Likewise, the increased representation of several Gram-negative bacteria was less pronounced than in the rats treated with vancomycin. However, the changes induced by the combination of naproxen and kanamycin were not markedly different from the changes induced by kanamycin alone. Moreover, there was no apparent correlation between the observed

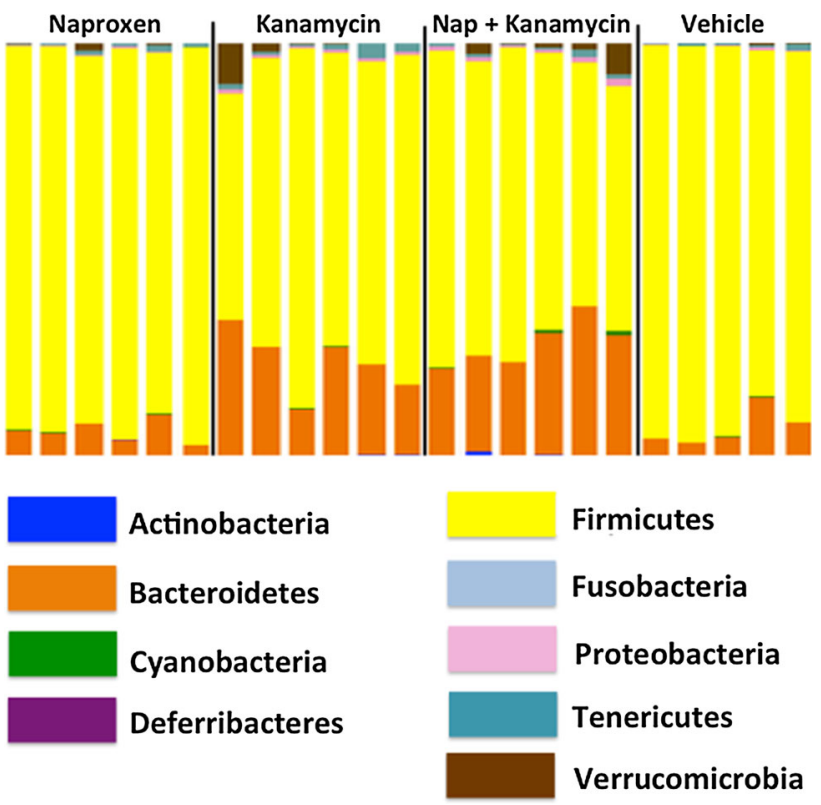

Fig. 4 Treatment with naproxen $(20 \mathrm{mg} / \mathrm{kg})$ and kanamycin $(100 \mathrm{mg} / \mathrm{kg})$ caused significant shifts in the microbiome of rats. Groups of 11-12 rats were treated orally with vehicle or kanamycin for a total of 9 days. Subgroups of five or six rats were treated orally with naproxen or vehicle twice daily for the final 4 days. 16S RNA Illumina sequencing of cecal samples from each rat was performed. Each vertical bar represents the sequencing data for one rat. Treatment with vehicle or kanamycin alone did not elicit any small intestinal damage. In contrast, treatment with naproxen plus vehicle or naproxen plus kanamycin resulted in extensive ulceration in the small intestine in the two groups (mean damage scores of 120 and 212, respectively; not significantly different; see Fig. 1b). Nap naproxen 
changes in the microbiota, and the extent of small intestinal damage that was observed (see Fig. 1b).

\section{Can probiotics protect against NSAID enteropathy?}

Our studies demonstrating that PPI-induced changes in the rat microbiome were responsible for the observed increase in susceptibility to NSAID enteropathy included the observation that replenishment of intestinal levels of Bifidobacterium restored resistance to NSAID enteropathy [13]. Several studies have demonstrated beneficial effects of probiotics, including Lactobacillus acidophilus and Lactobacillus casei, in experimental models of NSAID enteropathy $[30,35]$. There have also been human studies demonstrating preventative effects of a single probiotic $(L$. casei) [36] or of a cocktail of probiotics [37] against NSAID-induced small intestinal injury. We conducted a series of studies to attempt to confirm these previous findings, and to explore possible mechanisms of action.

As shown in Fig. 5a, administration of Bifidobacterium adolescentis once daily for 5 days prior to initiation of twice daily naproxen administration resulted in a substantial reduction of intestinal ulceration. However, not all Bifidobacterium species are effective for this application. For example, Bifidobacterium longum subsp. longum JCM 1217 was ineffective in the same model.

\section{Do substances released from probiotics account for their beneficial effects?}

Colonization of ulcers by bacteria may not be necessary to affect ulcer formation or healing. Of course, endotoxin liberated from bacteria can exacerbate ulceration and delay healing [31]. It is also possible that metabolites from bacteria can influence these processes. For example, Watanabe et al. [35] highlighted the possibility that lactic acid released by $L$. casei strain Shirota might contribute to the ability of that probiotic to reduce the severity of indomethacin-induced intestinal damage in rats. Kinouchi et al. [30] demonstrated that supernatants from cultures of $L$. acidophilus or B. adolescentis, but not the bacteria themselves, could significantly reduce NSAID-induced intestinal damage.

We performed studies to further examine the possibility that bacterial products, such as lactic acid, could prevent NSAID enteropathy. We compared the effects of a number of strains of Bifidobacterium, because a study by Fukuda et al. [38] had demonstrated differences in their ability to protect against enteropathogenic Escherichia coli infection in mice. Fukuda et al. attributed protection by some Bifidobacterium strains to their production of acetate, a short- chain fatty acid. Short-chain fatty acids are products of colonic fermentation, and have been associated with a reduced risk of many conditions, including inflammatory bowel disease, irritable bowel syndrome, cancer, and cardiovascular disease [39].

In addition to the Bifidobacterium species that we used (Fig. 5a), Fukuda et al. [38] used two other bacteria: $B$. longum subsp. longum NCC 1205 and a mutant of $B$. longum subsp. longum NCC 1205 in which a gene involved in acetate production was deleted via homologous recombination. In vitro metabolic profiling had confirmed that the bacteria that were effective in preventing enterohemorrhagic E. coli infection had higher rates of fructose consumption and acetate production [38]. Thus, B. longum subsp. longum JCM 1217 and $B$. longum subsp. longum NCC 1205 are high producers of acetate, $B$. adolescentis is an intermediate producer of acetate, and B. longum subsp. longum NCC 1205 mutant does not produce any acetate. As shown in Fig. 5b, both B. longum subsp. longum NCC 1205 and its mutant substantially reduced the severity of naproxen-induced small intestinal ulceration, to a similar extent as was observed with B. adolescentis. These observations strongly suggest that acetate production was not the underlying mechanism for the protective effects of these probiotics.

To investigate further the role of short-chain fatty acids in our model, we treated naproxen-treated rats with the commensal bacterium Faecalibacterium prausnitzii prior to naproxen administration. $F$. prausnitzii is an acetate consumer and butyrate producer [40]. $F$. prausnitzii has been identified as an important contributor to human health [41] and as a clinically significant protective factor against relapses of Crohn's disease [42]. Administration of $F$. prausnitzii for several days prior to beginning twice daily treatment with naproxen resulted in a marked reduction in the severity of intestinal damage (Fig. 5c). However, administration of the supernatant of $F$. prausnitzii had no effect on the severity of naproxen-induced intestinal damage.

\section{Future perspectives}

A review of the literature suggests that antibiotics that target Gram-negative bacteria are usually effective in reducing the extent of NSAID-induced intestinal ulceration $[28,31,32]$. On the other hand, antibiotics that target Gram-positive bacteria do not appear to alter the severity of NSAID-induced intestinal damage [31]. However, there are two major limitations to most of the existing literature evaluating the co-administration of antibiotics and NSAIDs. First, these studies often involved administration of only a single, large dose of an NSAID to the rodents, which 


\section{A}

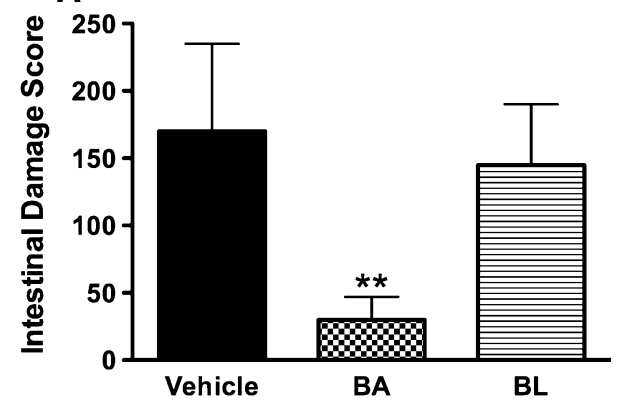

B
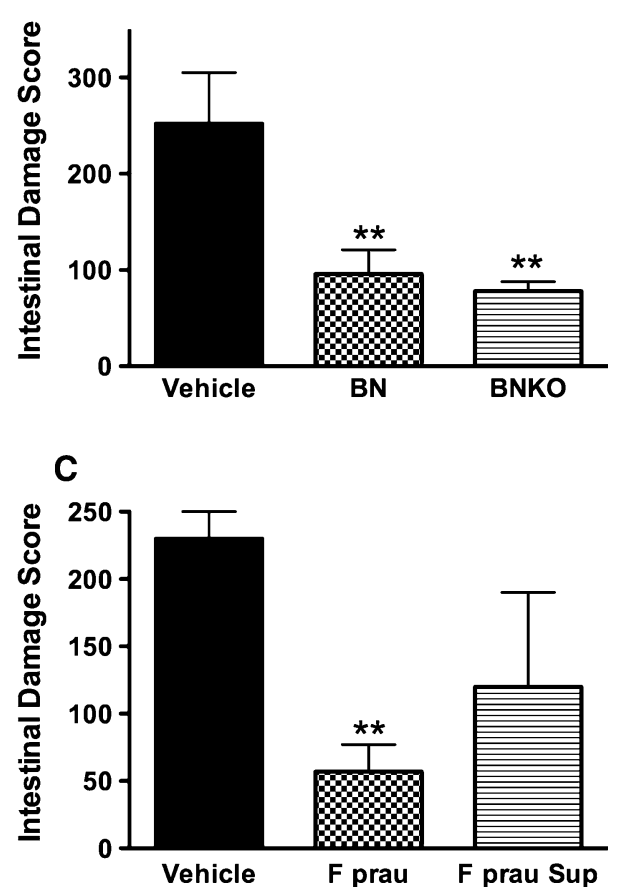

Fig. 5 Effects of various bacteria on the severity of naproxeninduced small intestinal damage. a When administered to rats once daily for 5 days prior to initiation of twice daily treatment with naproxen, Bifidobacterium adolescentis $(B A)$ significantly reduced the severity of intestinal damage. In contrast, treatment with Bifidobacterium longum subsp. longum JCM $1217(B L)$ had no effect on the severity of naproxen-induced intestinal damage. Each bar represents the mean \pm the standard error of the mean (SEM) of at least nine rats per group. b Treatment (as for a) with B. longum subsp. longum NCC $1205(B N)$ or a mutant of this in which a gene involved in acetate production was deleted via homologous recombination (BNKO) significantly reduced the severity of naproxen-induced intestinal damage. Each bar represents the mean \pm SEM of at least six rats per group. c Treatment (as for a) with Faecalibacterium prausnitzii ( $F$ prau) significantly decreased the severity of intestinal damage, whereas the supernatant from cultures of $F$. prausnitzii ( $F$ prau Sup) had no significant effect. Each bar represents the mean \pm SEM of at least four rats per group. ${ }^{*} p<0.01$ versus the vehicle-treated group (one-way ANOVA and Dunnett's multiple comparison test)

does not adequately represent the clinical scenario of longterm NSAID administration. Second, most studies have used indomethacin, an NSAID that is exceptionally damaging to the intestine and that is therefore rarely used for long-term treatment.

Although an abundance of evidence exists to suggest that bacteria contribute to the development of NSAID enteropathy, whether the bacteria directly initiate the damage once the intestine has been rendered susceptible to injury through inhibition of mucosal prostaglandin synthesis or whether bacteria exacerbate pre-existing tissue injury and impede repair remains unclear [1,2]. An additional layer of complexity in determining the role of bacteria in NSAID enteropathy is that the intestinal microbiota, enterohepatic circulation, and bile are all closely interrelated. Thus, multiple variables could influence the resistance to intestinal ulcerations observed in germfree rodents, or rodents with a ligated bile duct, after NSAID administration. For instance, the ligation of the bile duct prevents both the enterohepatic circulation of the NSAID and the entry of bile acids (luminal irritants) into the small intestine. In germ-free rodents, enterohepatic circulation of NSAIDs does not occur, as there is no bacterial $\beta$-glucuronidase present to cleave the NSAID from the glucuronide group, thereby allowing reabsorption [43]. Another variable that may influence the resistance of germfree rodents to intestinal damage is that they do not exhibit any secondary bile acids, because of the absence of bacterial enzymes that convert primary bile acids to secondary bile acids $[19,44]$.

Cocktails of antibiotics can reduce the severity of NSAID enteropathy, but they cannot completely prevent the damage. Some antibiotics, such as vancomycin, can cause dramatic changes in the microbiota (shift from primarily Gram-positive bacteria to primarily Gram-negative bacteria), but this did not result in intestinal damage. This strongly suggests that bacteria play a secondary role in the pathogenesis of NSAID enteropathy.

Acknowledgments We thank Webb McKnight for technical assistance. This work was supported by research grants from Crohn's Colitis Canada and from the Canadian Institutes of Health Research.

Conflict of interest The authors declare that they have no conflict of interest.

\section{References}

1. Wallace JL. NSAID gastropathy and enteropathy: distinct pathogenesis likely necessitates distinct prevention strategies. Br J Pharmacol. 2012;165:67-74.

2. Wallace JL. Mechanisms, prevention and clinical implications of nonsteroidal anti-inflammatory drug-enteropathy. World J Gastroenterol. 2013;19:1861-76.

3. Kearney PM, Baigent C, Godwin J, et al. Do selective cyclooxygenase-2 inhibitors and traditional non-steroidal anti-inflammatory drugs increase the risk of atherothrombosis? Meta-analysis of randomized trials. Br Med J. 2006;332:1302-8. 
4. Lanas A, Sopena F. Nonsteroidal anti-inflammatory drugs and lower gastrointestinal complications. Gastroenterol Clin North Am. 2009;38:333-52.

5. Wallace JL. Polypharmacy of osteoarthritis: the perfect intestinal storm. Dig Dis Sci. 2013;58:3088-93.

6. Maiden L, Thjodleifsson B, Theodors A, et al. A quantitative analysis of NSAID-induced small bowel pathology by capsule enteroscopy. Gastroenterology. 2005;128:1172-8.

7. Graham DY, Opekun AR, Willingham FF, Qureshi WA. Visible small-intestinal mucosal injury in chronic NSAID users. Clin Gastroenterol Hepatol. 2005;3:55-9.

8. Watanabe T, Tanigawa T, Nadatani Y, et al. Risk factors for severe nonsteroidal anti-inflammatory drug-induced small intestinal damage. Dig Liver Dis. 2013;45:390-5.

9. Endo H, Sakai E, Higurashi T, et al. Differences in the severity of small bowel mucosal injury based on the type of aspirin as evaluated by capsule endoscopy. Dig Liver Dis. 2012;44:833-8.

10. McCarthy DM. GI bleeding: problems that persist. Gastrointest Endosc. 2009;70:225-8.

11. Lanas A, García-Rodríguez LA, Polo-Tomás M, et al. Time trends and impact of upper and lower gastrointestinal bleeding and perforation in clinical practice. Am J Gastroenterol. 2009; 104:1633-41.

12. Bombardier C, Laine L, Reicin A, et al. A comparison of upper gastrointestinal toxicity of rofecoxib and naproxen in patients with rheumatoid arthritis. N Engl J Med. 2000;343:1520-8.

13. Wallace JL, Syer S, Denou E, et al. Proton pump inhibitors exacerbate NSAID-induced small intestinal injury by inducing dysbiosis. Gastroenterology. 2011;141:1314-22.

14. Satoh H, Amagase K, Takeuchi K. Exacerbation of non- steroidal anti-inflammatory drug-induced small intestinal lesions by antisecretory drugs in rats: the role of intestinal motility. J Pharmacol Exp Ther. 2012;343:270-7.

15. Blackler R, Syer S, Bolla M, et al. Gastrointestinal-sparing effects of novel NSAIDs in rats with compromised mucosal defence. PLoS One. 2012;7:e35196.

16. Blackler RW, Gemici B, Manko A, Wallace JL. NSAID-enteropathy: new aspects of pathogenesis and prevention. Curr Opin Pharmacol. 2014;19C:11-6.

17. Elliot SN, Buret A, McKnight W, Miller MJ, Wallace JL. Bacteria rapidly colonize and modulate healing of gastric ulcers in rats. Am J Physiol. 1998;275:G425-32.

18. Martinez-Augustin O, Sanchez de Medina F. Intestinal bile acid physiology and pathophysiology. World J Gastroenterol. 2008;14:5630-40.

19. Duboc H, Rajca S, Rainteau D, et al. Connecting dysbiosis, bileacid dysmetabolism and gut inflammation in inflammatory bowel diseases. Gut. 2013;62:531-9.

20. Blackler RW, Motta JP, Manko A, et al. Hydrogen sulphide protects against NSAID-enteropathy through modulation of bile and the microbiota. Br J Pharmacol. doi:10.1111/bph.12961.

21. Somasundaram S, Rafi S, Hayllar J, et al. Mitochondrial damage: a possible mechanism of the "topical" phase of NSAID induced injury to the rat intestine. Gut. 1997;41:344-53.

22. Brune K, Dietzel K, Nurnberg B, Schneider HT. Recent insight into the mechanism of gastrointestinal tract ulceration. Eur $\mathrm{J}$ Rheumatol Inflamm. 1987;9:8-14.

23. Kent TH, Cardelli RM, Stamler FW. Small intestinal ulcers and intestinal flora in rats given indomethacin. Am $\mathrm{J}$ Pathol. 1969;54:237-49.

24. Wax J, Clinger WA, Varner P, et al. Relationship of the enterohepatic cycle to ulcerogenesis in the rat small bowel with flufenamic acid. Gastroenterology. 1970;58:772-80.

25. Reuter BK, Davies NM, Wallace JL. Nonsteroidal anti- inflammatory drug enteropathy in rats: role of permeability, bacteria, and enterohepatic circulation. Gastroenterology. 1997;112:109-17.
26. LoGuidice A, Wallace BD, Bendel L, et al. Pharmacologic targeting of bacterial $\beta$-glucuronidase alleviates nonsteroidal antiinflammatory drug induced enteropathy in mice. J Pharmacol Exp Ther. 2012;341:447-54.

27. Robert A, Asano T. Resistance of germ-free rats to indomethacininduced intestinal lesions. Prostaglandins. 1977;14:333-41.

28. Uejima M, Kinouchi T, Kataoka K, et al. Role of intestinal bacteria in ileal ulcer formation in rats treated with a nonsteroidal antiinflammatory drug. Microbiol Immunol. 1996;40:553-60.

29. Bjarnason I, Hayllar J, Smethurst P, et al. Metronidazole reduces intestinal inflammation and blood loss in non-steroidal antiinflammatory drug induced enteropathy. Gut. 1992;33:1204-8.

30. Kinouchi T, Kataoka K, Bing SR, et al. Culture supernatants of Lactobacillus acidophilus and Bifidobacterium adolescentis repress ileal ulcer formation in rats treated with a nonsteroidal antiinflammatory drug by suppressing unbalanced growth of aerobic bacteria and lipid peroxidation. Microbiol Immunol. 1998;42:347-55.

31. Watanabe T, Higuchi K, Kobata A, et al. Non-steroidal antiinflammatory drug-induced small intestinal damage is Toll-like receptor 4 dependent. Gut. 2008;57:181-7.

32. Koga J, Aoyagi K, Matsumoto T, et al. Experimental enteropathy in athymic and euthymic rats: synergistic role of lipopolysaccharide and indomethacin. Am J Physiol. 1999;276:G576-82.

33. Leite AZ, Sipahi AM, Damiao AO, et al. Protective effect of metronidazole on uncoupling mitochondrial oxidative phosphorylation induced by NSAID: a new mechanism. Gut. 2001;48:163-7.

34. Bartram AK, Lynch MD, Stearns JC, et al. Generation of multimillion-sequence 16S rRNA gene libraries from complex microbial communities by assembling paired-end illumina reads. Appl Environ Microbiol. 2011;77:3846-52.

35. Watanabe T, Nishio H, Tanigawa T, et al. Probiotic Lactobacillus casei strain Shirota prevents indomethacin-induced small intestinal injury: involvement of lactic acid. Am J Physiol Gastrointest Liver Physiol. 2009;297:G506-13.

36. Endo H, Higurashi T, Hosono K, et al. Efficacy of Lactobacillus casei treatment on small bowel injury in chronic low-dose aspirin users: a pilot randomized controlled study. J Gastroenterol. 2011;46:894-905.

37. Gallo A, Curigliano V, et al. Clinical trial: the effects of a probiotic mixture on non-steroidal anti- inflammatory drug enteropathy - a randomized, double- blind, cross-over, placebocontrolled study. Aliment Pharmacol Ther. 2010;32:209-14.

38. Fukuda S, Toh $\mathrm{H}$, Hase K, et al. Bifidobacteria can protect from enteropathogenic infection through production of acetate. Nature. 2011;469:543-7.

39. Hijova E, Chmelarova A. Short chain fatty acids and colonic health. Bratisl Lek Listy. 2007;108:354-8.

40. Duncan SH, Hold GL, Harmsen HJ, et al. Growth requirements and fermentation products of fusobacterium prausnitzii, and a proposal to reclassify it as Faecalibacterium prausnitzii gen. nov., comb. nov. Int J Syst Evol Microbiol. 2002;52:2141-6.

41. Miquel S, Martín R, Rossi O, et al. Faecalibacterium prasnitzii and human intestinal health. Curr Opin Microbiol. 2013;16:255-61.

42. Sokol H, Pigneur B, Watterlot L, et al. Faecalibacterium prausnitzii is an anti-inflammatory commensal bacterium identified by gut microbiota analysis of Crohn disease patients. Proc Natl Acad Sci U S A. 2008;105:16731-6.

43. Treinen-Moslen M, Kanz MF. Intestinal tract injury by drugs: importance of metabolite delivery by yellow bile road. Pharmacol Ther. 2006;112:649-67.

44. Begley M, Gahan CG, Hill C. The interaction between bacteria and bile. FEMS Microbiol Rev. 2005;29:625-51. 